\title{
Elevated liver function tests in a coin collector
}

\author{
Mukhamad Sami Valid* \\ Locum Tenens Hospitalist, USA
}

\section{Introduction}

Liver is a vital organ; without it life is impossible. Abnormalities in liver function tests are frequently encountered in everyday medical practice. In some cases, this can present a clinical puzzle that requires brainstorming and multiple consultations. We present an interesting case with impressive clinical picture and benign outcome.

\section{Case report}

A 21 year old man of thin body habitus with history of diabetes mellitus type 1 and celiac disease presented to the Emergency Room after midnight with complaint of right upper abdominal pain of achy character and medium intensity without radiation that bothered him on and off for weeks-months but worsened earlier in the day. $\mathrm{He}$ denied fever, chills, nausea, vomiting, or change in stool. Labs showed elevated liver function tests (LFTs). CT showed hepatomegaly, $25 \mathrm{~cm}$ in midclavicular line. The case was passed to the hospitalist for further evaluation. Upon further interview and questioning about hobbies and contact with chemicals the patient said he collected coins and cleaned them with acetone. Upon further inquiry he admitted he used acetone with bare hands and leaved it open in closed environment. The other compound he admitted to use was EZest (sulphuric acid and thiourea). He said he cleaned his coins 2-3 times a week, 1-2 hours at a time. Coins were made of silver, copper, and nickel. The patient was admitted to the Medical Floor and placed on nothing by mouth (NPO) and IV hydration with normal saline $100 \mathrm{ml} / \mathrm{h}$. The next day toxicology consult was done and more tests were ordered.

The following lab results were obtained:

Alk Phos 232 dropped to 178 the next day

ALT 919 dropped to 542 the next day

AST 1155 dropped to 226 the next day

Bilirubin total 0.3 and remained at the same level

Albumin 3.1 on admission dropped to 2.7 the next day

Protein 7.2 dropped to 6 the next day

Urine drug screen positive for THC

Hepatitis profile A Ab Igm, B surface antigen, B core antigen, C $\mathrm{AB}$, nonreactive

Infectious mononucleosis negative

Iron 127

Ferritin 186

Transferrin 305

Transferrin saturation $30 \%$

\author{
Copper 138 \\ Ceruloplasmin 35 \\ Lead $<2$ \\ Acetone $<2$ \\ Methanol $<5$ \\ Isopropanol $<5$ \\ Mercury $<3$ \\ Ethanol undetectable \\ Acetaminophen $<1$ \\ TSH 3.75 \\ HIV $1 \& 2$ AB negative \\ Tissue transglutaminase 1 \\ Creatinine 1 \\ Glucose 218 \\ HbA1c 12.5 \\ Lipase 45 \\ UA glucose $>500$
}

No clear reason for abnormal LFTs was named. LFTs improved quickly and the patient was discharged home. He was scheduled to follow up with hepatologist and in case LFTs do not return to normal liver biopsy is indicated.

\section{Discussion}

Acetone is a clear liquid, volatile, and inflammable. Hundreds of commonly used household products contain acetone, including furniture polish, rubbing alcohol, and nail polish remover. Acetone poisoning is rare [1]. The organism is capable of breaking down large amounts of acetone naturally. However, poisoning can occur by exposure to very large amounts within a short period of time or smaller quantities over an extended period of time.

Toxicology consult mentioned that acetone is not known to have active hepatotoxic effects but can increase toxic effects from trichloroethylene, tetrahydrocarbons, haloalkanes and other

Correspondence to: Mukhamad Sami Valid, Locum Tenens Hospitalist, USA, E-mail: mswalid@yahoo.com

Received: October 11, 2017; Accepted: November 01, 2017; Published: November 03, 2017 


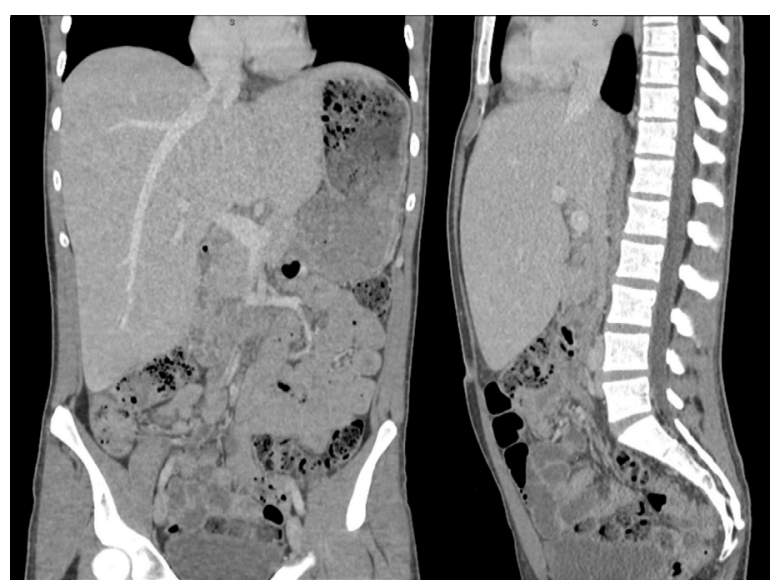

Figure 1. Coronal section of CT abdomen-pelvis and Anterior-posterior section of CT abdomen-pelvis.

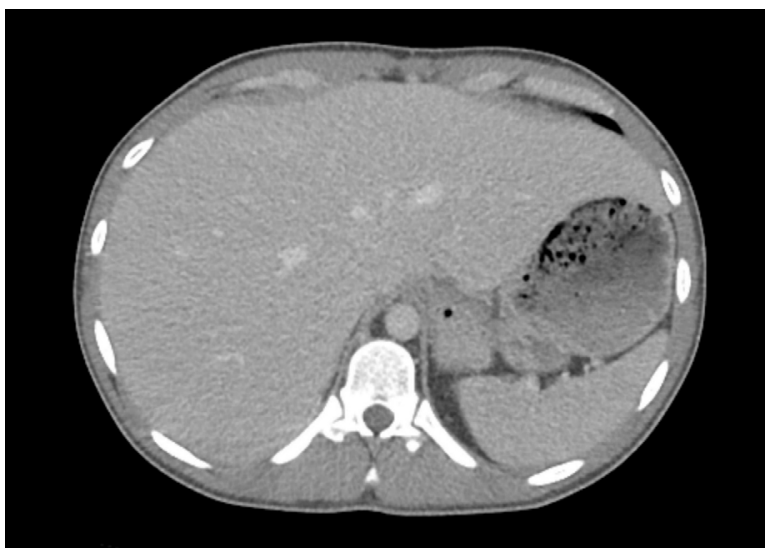

Figure 2. Trasverse section of CT abdomen-pelvis. compounds [2-6]. It also may liberate polyvinyl chloride residues which have liver disease and carcinogenic effects [7]. Other suggested possibilities were heavy metal exposure or autoimmune hepatitis. However, the fleeting nature of the rise in LFTs and quick improvement in hospital away from acetone exposure probably favors the theory of a chemical toxic effect on liver.

\section{Conclusion}

Liver damage has been linked to certain professions such as shoe repair [8]. A useful differential diagnosis information can be learnt from our case report is that the hobby of coin collecting may be associated with increased risk of abnormal LFTs.

\section{References}

1. Mirchev H (1978) Hepatorenal lesions in acute acetone poisoning. Vutr Boles 17: 8992. [Crossref]

2. MacDonald JR, Gandolfi AJ, Sipes IG (1982) Acetone potentiation of 1,1,2-trichloroethane hepatotoxicity. Toxicol Lett 13: 57-69. [Crossref]

3. Hewitt WR, Brown EM, Plaa GL (1983) Acetone-induced potentiation of trihalomethane toxicity in male rats. Toxicol Lett 16: 285-296. [Crossref]

4. Hewitt WR, Plaa GL (1983) Dose-dependent modification of 1,1-dichloroethylene toxicity by acetone. Toxicol Lett 16: 145-152. [Crossref]

5. Hewitt WR, Miyajima H, Côté MG, Plaa GL (1980) Modification of haloalkaneinduced hepatotoxicity by exogenous ketones and metabolic ketosis. Fed Proc 39: 3118-3123. [Crossref]

6. Traiger GJ, Plaa GL (1974) Chlorinated hydrocarbon toxicity. Potentiation by isopropyl alcohol and acetone. Arch Environ Health 28: 276-278. [Crossref]

7. Creech JL, Makk L (1975) Liver disease among polyvinyl chloride production workers Ann N Y Acad Sci 246: 88-94. [Crossref]

8. Tomei F, Giuntoli P, Biagi M, Baccolo TP, Tomao E, et al. (1999) Liver damage among shoe repairers. Am J Ind Med 36: 541-547. [Crossref]

Copyright: $(2017$ Valid MS. This is an open-access article distributed under the terms of the Creative Commons Attribution License, which permits unrestricted use, distribution, and reproduction in any medium, provided the original author and source are credited. 